\title{
Improved detection of metastases by color Doppler ultrasonography
}

\author{
Petar Avramovski ${ }^{1 *}$, Maja Avramovska ${ }^{2}$ and Emilija Sikole ${ }^{3}$ \\ ${ }^{1}$ Clinical Hospital "D-r Trifun Panovski” Department of internal medicine, Partizanska bb. 7000 Bitola, Macedonia. Professor of High Medical School and Faculty \\ of Veterinary Medicine, St. Clement of Ohrid University- Bitola, Partizanska bb. 7000 Bitola, Macedonia \\ ${ }^{2}$ University Clinic of Obstetrics and Gynecology, Specializing at University "SS. Cyril and Methodius", Vodnjanska 17, 1000, Skopje, Macedonia \\ ${ }^{3}$ Institute of Preclinical and Clinical Pharmacology with Toxicology, University "SS. Cyril and Methodius”, Vodnjanska 17, 1000, Skopje, Macedonia
}

\section{Introduction}

Metastases are the most common malignant liver lesion and the most indication for B-mode ultrasonography. Hepatic metastases are 14-40 times more common than primary liver tumors [1]. One of the main difficulties in liver imaging for metastatic disease is the high prevalence of benign liver lesion that can be misinterpreted as evidence of metastatic disease. Liver haemangiomas, focal nodular hyperplasia and additionally, pseudolesions (transient hepatic attenuation differences, focal fatty sparing/focal fatty change) are the main sources of confusion [2].

It can be very hard to localize focal liver lesions and metastasis. Also, could be very difficult to distinguish isoechoic nodules and metastasis from adjacent liver tissue $[3,4]$. Ultrasound is quick, inexpensive, safe and easy to perform procedura, but color Doppler ultrasound can provide additional information regarding vascularity of the lesion [4]. In general, however, metastases may appear as rounded and well defined, hypoechoic, positive mass effect with distortion of adjacent vessels, hypoechoic halo due to compressed and fat spared liver, cystic, calcified, infiltrative and echogenic appearance are all possible $[1,2]$.

\section{Doppler ultrasonography characteristics of focal liver disease}

The liver is unusual in that it has a dual vascularization, functional and nutritional bloodstream organized through a system of a hepatic artery and a portal vein, respectively. Most normal liver cells are fed by branches of the portal vein, whereas cancer cells in the liver are usually fed by branches of the hepatic artery [5]. Color Doppler ultrasonography allows easy detection of blood vessels, the speed and direction of blood stream, volume, and wave form of flow, whether pulsating or continuous, can be determined.

In tumors or tumor-like lesion, we can detect three or four different types of color Doppler signals:

1. A feeding signal with a continuous or pulsating wave. This signal goes into the lesion from the outside (Figure 1).

2. A Doppler signal with continuous or pulsating wave which is detected in lesion as spotty or short linear signals (Figure 2).

3. The third is a drainage signal with continuous wave which goes from the inside to the outside of the lesion (Figure 3).

4. A penetrating signal with a pulsating or continuous wave which penetrates all way through the lesion. The penetrating signals are detected in metastasis but not in hepatocellular carcinoma or hemangoma [6].

\section{Images interpretation}

\section{Feeding signals}

The color Doppler ultrasound image presented by figure 1 showed hepatocelular carcinoma (HCC) complicated with hepatic cirrhosis (autopsy proven), with emboli in the portal vein detected previously by conventional B-mode ultrasonography. The rounded lesion with hypoechoic "halo" largest in diameter and localized in the left part of the image presented as HCC. The smaller hypoechoic lesions are secondary metastatic deposits. Color Doppler arbitrarily displays blood flow toward the transducer as red and it is generated by portal vein blood flow. The blood flow away from the transducer

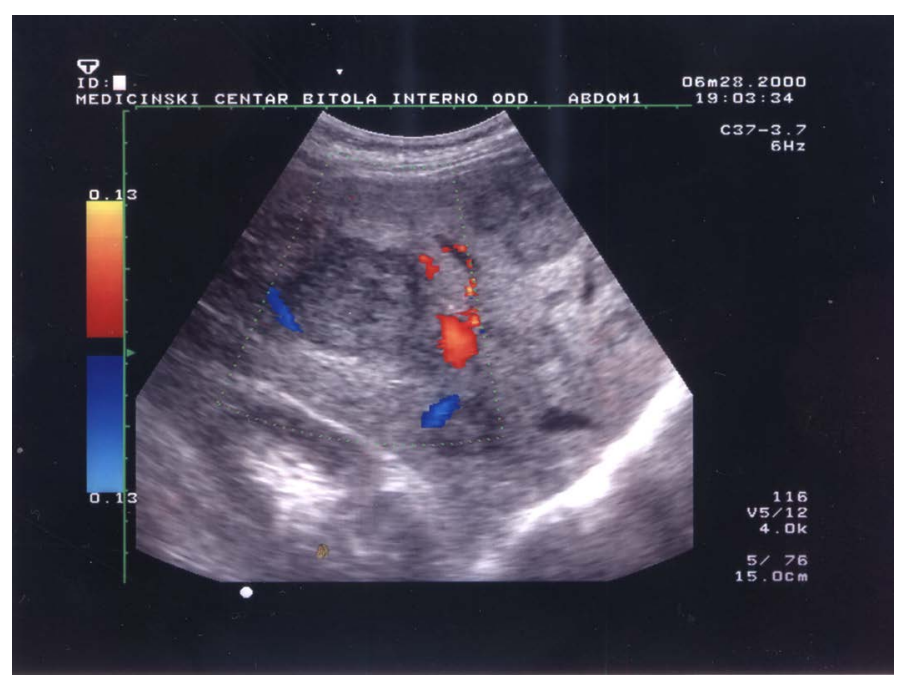

Figure 1. Image of iso/hypoechoic round, heterogeneous and moderately well-defined lesion showing the vessel that feeds the lesion (hepatocelular carcinoma).

Correspondence to: Petar Avramovski, Clinical Hospital "D-r Trifun Panovski” Department of internal medicine, Partizanska bb. 7000 Bitola, Macedonia. Professor of High Medical School and Faculty of Veterinary Medicine, St. Clement of Ohrid University- Bitola, Partizanska bb. 7000 Bitola, Macedonia, Tel. +389-70-207-187, E-mail: avramovski@gmail.com

Received: May 21, 2017; Accepted: June 09, 2017; Published: June 12, 2017 


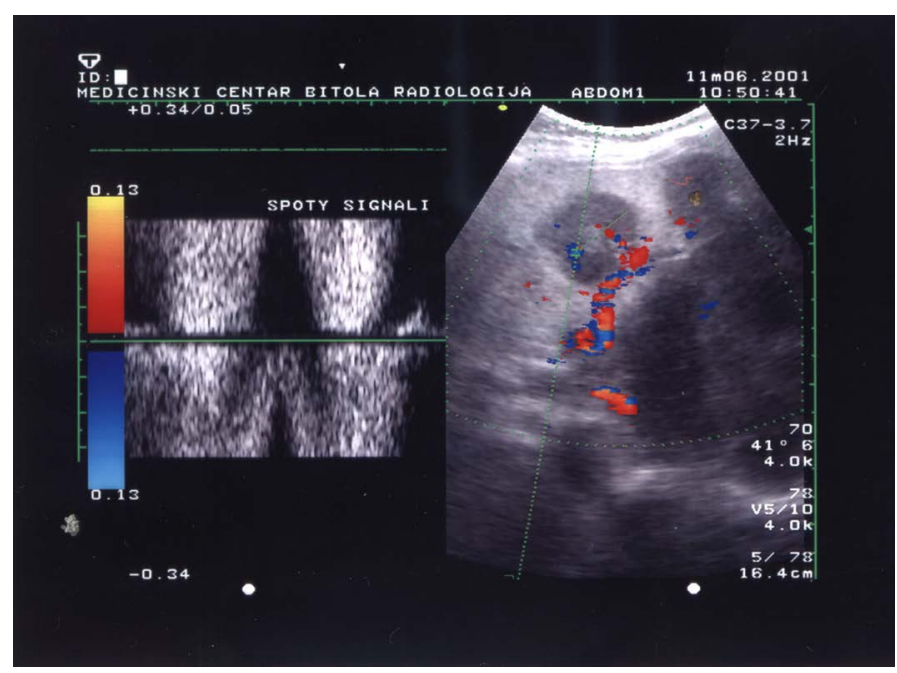

Figure 2. Doppler flow wave-form inside of metastasis vascularisation showed spotty signals.

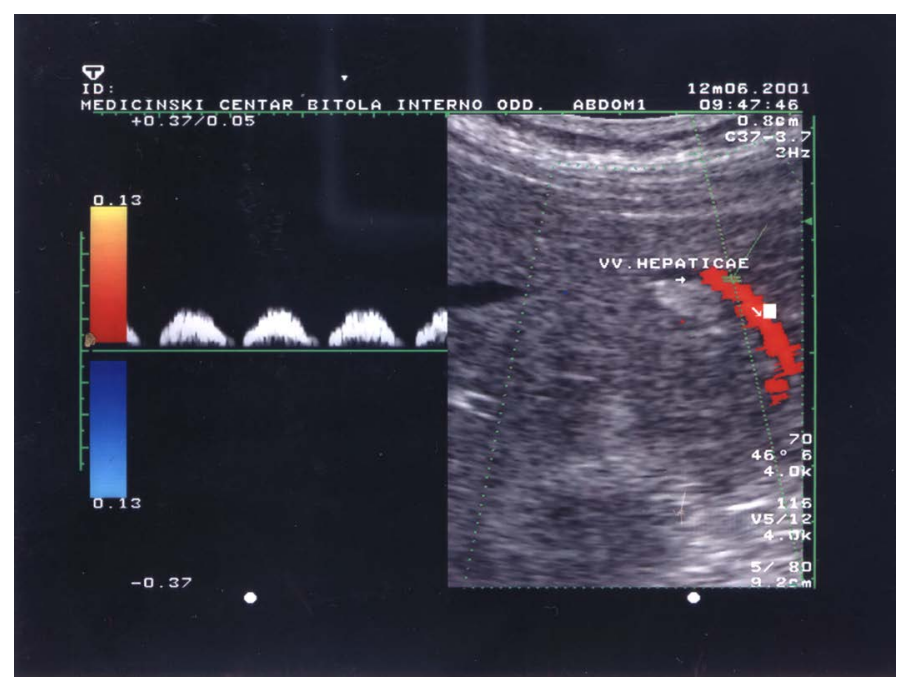

Figure 3. Image of hyperechoic heterogeneous lesson with anechoic "halo" and flow specter of portal blood drainage.

as blue and it is belonging to hepatic vein flow. Sometimes, we used the terms antegrade and retrograde to describe flow in this context. At spectral Doppler blood flow (angiodinogram) toward the transducer is displayed above the baseline (antegrade flow) and blood flow away from the transducer is displayed below the baseline (retrograde flow) [7]. The branch of hepatic vein presented as blue vessel in 8-9 a clock position, superficially incorporated in HCC is the feeding vessel. The small red colored crescent area in 2 a clock position is presented by hepatic artery.

Halo in liver metastases correspond to the vascular flow [8,9], but is not present in this image. Only Doppler examination with properly administrated contrast-enhanced agent Levovist can show the flow in the halo. The detection of peripheral halo flow is improved by power Doppler instead a conventional color Doppler, from 34\% to $77 \%$ detection rate. Use of contrast media enhanced the power Doppler detection rate to $98 \%(\mathrm{P}<0.005)[8]$.

\section{Spotty signals}

The signal received by irregular neovascular bloodstream, which do not have any regularity in the amplitudes, frequency or time duration of the propagation and incidentally appearing in chaotic time continuum, independently of phase of cardiac cycle (systole and diastole) are called chaotic or spotty signals. Signals like these, have not characteristic to any known vascular bloodstream, and do not look like any of the vein or arterial blood flow. Those are signals generated by the new-created blood vessels in the interior of the metastasis in the neoangiogenesis process.

The slow and chaotic spotty signals are possible for visualization only with correct manual filter settings on low frequency filter. Low flow gain results with missed signal and high flow gaing results with color signal of unexistable flow [3,9].

Figure 2 shows two metastases with central anechoic pattern. The sample volume was positioned in the central of the left metastasis. We used a peak repetition frequency (PRF) value of $4 \mathrm{KHz}$ and angle of insonation of $41^{\circ}$. Using a small PRF in high speed blood flow is incorrect and produces aliasing and other artefacts. In that case we can make incorrect diagnosis for some metastasis, a haemangioma and other nonvascular structure. High PRF reduces the depth of penetration of ultrasound signals [3]. The finding of a central anechogenic area and peripheral vascularity, as well as signs of invasion into neighboring blood vessels, is aditional indications of a malignancy [10].

\section{Drainage signals}

Hepatocellular carcinoma is supposed to have a venous drainage system to a portal vein, which makes intrahepatic metastasis possible [11]. The image 3 shows HCC in initial stage with hyperechoic heterogeneous sonographic expression and anechoic "halo". The red colored vascular area shows portal venous invasion, but neither showed microscopic hepatic venous invasion. Low speed blood flow in hepatic vein (see marked part of the figure 3 with white small arrow) and relatively high value of selected $\mathrm{PRF}(4 \mathrm{KHz})$ do not give possibility to color the vessel.

The sample volume marker is positioned inside portal vein to show portal flow specter of drainage vessel. The isoechoic oval area below the hyperechoic HCC is regenerative nodule on altered cirrhotic liver parenchyma. The specter of drain aging portal vessel is typical pulsatile portal flow pattern with flattened curve and high pulsatility presented as an intermittent zero flow between end of the diastole and beginning of the systole.

Mitsunobu, et al. demonstrated that the portal vein serves as an efferent vessel in advanced HCC by direct injection of radiopaque media into HCC nodules of resected specimens [11,12]. They found that tumor spread in HCC progresses from capsular invasion to intrahepatic invasion and that the portal vein may act as an efferent tumor vessel. In figure 3 we present the portal vein which is red colored tube, as an efferent HCC vessel.

\section{Non-neoplastic hepatic lesion}

Hepatic haemagiomas, also known as hepatic venous malformations, are benign non-neoplastic and hypervascular liver lesions. The most common benign hypervascular liver tumor requiring differential diagnosis is hemangioma [13]. From a clinical perspective, it is important to differentiate haemangiomas from hepatic neoplasms. In $98 \%$ from hepatic hemangiomas color or power Doppler imaging shows no internal blood flow. About half of those cases show a peripheral flow patter belives no represent flow in displaced blood vessels. Smal precent of hemagiomas $(<3 \%)$ that have unusual central fibrosis with large vessels shows diffusely increased blood flow on 


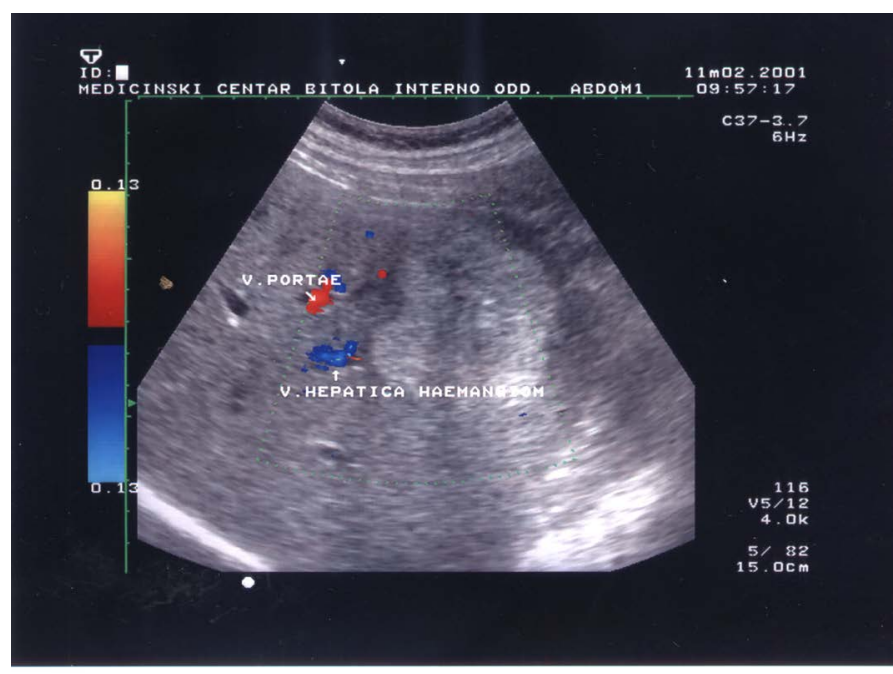

Figure 4. Image of non-vascular hepatic lesion, hepatic hemangioma.

power Doppler in peripheral vessels and internal vascularity as well [14]. Focal nodular hyperplasia (FNH), hepatic adenoma (HA) and hemangioma represent the most frequent non-vascular benign liver tumors. They are often asymptomatic [15].

Figure 4 shows hyperechogenic hepatic hemagioma. Flow is not evidences inside the lesion. In this female patient, aged 43 years, hemagiomas was associated by thrombocytopenia and hypofibrinogenemia which is called Kasabach-Merrit syndrome. The high echogenicity inside the hemangioma due to high percent of fatty deposits. This hemagioma belong to group of hepatic hemagiomas with fatty infiltration (biopsy approved).

\section{Conclusion}

The B-mode (gray-scale) ultrasound should not be abandoned as method of estimation of focal liver estimation. It have to be extended by Duplex technique and color Doppler ultrasound. Conventional blood flow velocity measurement in periphery and at the center of focal liver lesions is not precise and reliable enough to make real distinguish between benign and malignant liver lesions.

During metastatic and HCC lesions we can trust in some reliable criteria for detection and differentiation to be specific for malignancy: a central spot detection, halo sign without detectable blood flow, vascularization of focal liver lesion, chaotic blood vessel architecture, detection of feeding, drainage and spotty signals etc. Therefore, application of color Doppler and Duplex ultrasound is highly recommended as a conventional, inexpensive, fast, reliable and noninvasive imaging method of first choice in focal liver disease.

\section{References}

1. Namasivayam S, Martin DR, Saini S (2007) Imaging of liver metastases: MRI. Cancer Imaging 7: 2-9. [Crossref]

2. Lencioni R, Cioni D, Bartolozzi C (2005) Focal liver lesions, detection, characterization, ablation. Springer Verlag.

3. Petar Avramovski (2009) Value of ultrasound machine settings optimization for better diagnosis of focal liver lesions. Maced J Med Sci 2: 149-152.

4. Semelka RC, Martin DR, Balci NC (2005) Focal lesions in normal liver. J Gastroenterol Hepatol 20: 1478-1487. [Crossref]

5. DuradoB, William C, Colleen D, Koerner YJ, Lichtenfeld L, et al. (2016) Embolization therapy for liver cancer. Amer Cancer soc 3: 7-9.

6. Takayoshi T, Haruo K, Masahiko O, Masao O, Yasuo E, et al. (1992) Primary liver cancer in Japan. Tokyo: Springer Japan 123-124.

7. McNaughton DA, Abu-Yousef MM (2011) Doppler US of the liver made simple. Radiographics 31: 161-188. [Crossref]

8. Rafaelsen SR, Solvig J (2004) Ultrasound imaging of flow patterns in liver metastases from colorectal cancer. Scand J Gastroenterol 39: 761-765. [Crossref]

9. Grigorov N, Nikolova S (1997) Klinichna Dopler ehografia. Sofia: Medicinsko izdatelstvo 94-95.

10. Scholbach T, Scholbach J, Krombach GA, Gagel B, Maneschi P, Di Martino E (2005) New method of dynamic color doppler signal quantification in metastatic lymph nodes compared to direct polarographic measurements of tissue oxygenation. Int J Cancer 114: 957-962. [Crossref]

11. Miyazaki K, Soyama A, Hidaka M, Hamaaki K, Yamanouchi K, et al. (2011) Ex vivo hepatic venography for hepatocellular carcinoma in livers explanted for liver transplantation. World J Surg Oncol 9: 111. [Crossref]

12. Mitsunobu M, Toyosaka A, Oriyama T, Okamoto E, Nakao N (1996) Intrahepatic metastases in hepatocelular carcinoma: the role of the portal vein as an efferent vessel. Clin Exp Metastasis 14: 520-529. [Crossref]

13. Murakami T, Tsurusaki M (2014) Hypervascular benign and malignant liver tumors that require differentiation from hepatocellular carcinoma: key points of imaging diagnosis. Liver Cancer 3: 85-96. [Crossref]

14. Perkins AB, Imam K, Smith WJ, Cronan JJ (2000) Color and power Doppler sonography of liver hemangiomas: a dream unfulfilled? J Clin Ultrasound 28: 159-165. [Crossref]

15. Maillette de Buy Wenniger L, Terpstra V, Beuers U (2010) Focal nodular hyperplasia and hepatic adenoma: epidemiology and pathology. Dig Surg 27: 24-31. [Crossref]

Copyright: (C2017 Avramovski P. This is an open-access article distributed under the terms of the Creative Commons Attribution License, which permits unrestricted use, distribution, and reproduction in any medium, provided the original author and source are credited. 